\title{
Reduction of Crystallographic Point Groups to Subgroups by Homogeneous Stress
}

\author{
H. S. Peiser and J. B. Wachtman, Jr.
}

(February 23, 1965)

\begin{abstract}
It is assumed that the symmetry operations possessed by a homogeneously stressed crystal will be those common to the crystal and to the stress. Application of stress either leaves a point group unaltered or lowers it to a subgroup. Any stress-induced minimum step of symmetry lowering can be caused by unaxial stress but three of the possible stressinduced compound steps of symmetry lowering require biaxial stress. The uniaxial or biaxial stress required for any stress-induced symmetry lowering is tabulated and stereograms are given for each of the minimum steps showing the relation of the remaining symmetry operations to the initial symmetry and showing the splitting of a set of initially equivalent general directions into inequivalent subsets. These stereograms provide a direct representation of the splitting of a general position in a crystal into inequivalent subsets for the symmorphic space groups and the latter are listed; the effect of glide planes and screw axes must be considered in the remaining space groups yet the stereograms still provide the correct pattern of splitting. Uses of the stereograms, for instance, to obtain the splitting of a special position, are described.
\end{abstract}

\section{Introduction}

The response of crystals to stress has been studied for many years; the conditions on elastic constants and piezoelectric constants imposed by crystal symmetry are given, for example, by $\mathrm{Nye}[1] .{ }^{1} \mathrm{He}$ has also discussed second order effects including those arising when a crystal is stressed and simultaneously subjected to a second influence such as a beam of light. The treatment proceeds by considering that the stressed crystal has only the symmetry elements common to the stress and to the unstressed crystal; the electro-optical coefficients permitted by symmetry are those appropriate to the point group of the stressed crystal. If this is of lower order than the point group of the unstressed crystal, finite values are permitted for some of the electro-optical coefficients which were previously zero. In an actual experiment it is easier to apply a uniaxial stress than one which is biaxial or triaxial. The subgroups which can be reached by any homogeneous stress have been classified [2] and organized in to a successive subgroup scheme [3]; we shall show by tabulating appropriate tensile stresses that all but three of these symmetry reductions can be induced by uniaxial stress. The other three symmetry reductions require biaxial stress.

The effect of stress on symmetry restrictions of a tensor property can thus be determined if the tensor property is one previously classified in terms of symmetry of unstressed crystals. There are, however, other properties which are influenced by the molecular symmetry of the crystal and which reflect only the state of a particular set of atoms of one type rather than an average over all atoms of this type;

${ }_{1}^{1}$ Figures in brackets indicate the literature references at the end of this paper. such properties may require more detailed consideration of the symmetry lowering caused by stress. For example, the excitation spectra of donors in a semiconductor such as silicon depend on the site symmetry of the donor. In the case of silicon the usual donor atoms occupy substitutional sites with the full point-group symmetry of the crystal; application of homogeneous stress changes the symmetry of all sites equally and leads to a single pattern of splitting of excitation spectra for a given stress [4]. The point symmetry of initially equivalent sites in a crystal may, however, change unequally under a given stress and more complex patterns of splitting of spectra should then occur. For example, sites with symmetry $m$ in a crystal of symmetry $\overline{3} \mathrm{~m}$ can be affected by uniaxial stress so that two out of every three sites lose all symmetry while the third retains the symmetry $\mathrm{m}$. The possibility of such unequal symmetry lowering and the circumstances under which it should occur can be seen from stereograms showing the original symmetry operations and the symmetry retained under stress. These considerations of unequal symmetry lowering should also apply to the observation of magnetic resonance in crystals under homogeneous stress [5]. A minimum step of stress-induced lowering of symmetry has previously been defined for space groups as one which leaves no stress-induced subgroup of the initial group which is also a supergroup of the final group and which is distinct from both [6]. The same definition is taken for point groups. One might suppose that such a minimum step might leave a distinct subgroup not reachable by stress which is a distinct supergroup of the final group. Detailed examination shows that no such crystallographic point groups occur. A complete set of stereograms for all crystal symmetries and all possible minimum steps of symmetry lowering is given here. 
These stereograms also show the inequivalences introduced into a set of general sites; the results for special sites follow from these. Any nonminimum step of symmetry lowering by stress can be analyzed in terms of successive minimum steps.

The stereograms and stress tables given here should also be useful in other fields such as internal friction and the morphology of crystals [3, 7, 8, 9].

The splitting of a set of sites in a crystal into inequivalent subsets under stress has also been treated in the context of space groups [10], the relation of the present point-group considerations to the space-group treatment will be covered in the discussion.

\section{Description of Stereograms}

Stereograms representing each of the 32 crystallographic point groups and their conventional symbols are given in the International Tables for X-ray Crystallography [11]. These tables also list all of the subgroups of each point group; only certain of these subgroups can be reached by the action of homogeneous stress. They have been listed [3] and a systematic method of deriving this list is now presented in the appendix. It is useful to show the symmetry operations retained in a stress-induced subgroup and the original symmetry operations on the same stereogram. The latter can be shown by the conventional symbols used by the International Tables [11] but the former require new symbols. Both the conventional symbols and the new symbols are illustrated and defined in figure 1.
The stereograms are also shown in figure 1; those for centrosymmetric point groups are given first, beginning with those of highest order. The crystallographic axes are not marked, but the $z$-axis of the initial point group is taken to be at the center (north pole) of the stereogram. For all crystal systems (other than triclinic for which no stereogram is shown because no proper stress-induced subgroup exists) the angle between $z$ and $y$ is $90^{\circ}$. Therefore, in all cases the $y$-axis must fall on the primitive (equator) so according to accepted convention we have chosen the horizontal axis with positive $y$ towards the right.

In some stereograms a second heading, inclined at an angle to the first, is given. When using these, as explained subsequently, "horizontal" is with respect to the heading used. A given point group sometimes occurs in more than one orientation as a stress-induced subgroup of an initial point group. Alternative settings may be equivalent (i.e., one is generated from another by symmetry operations of the initial point group) or inequivalent. A second, nonequivalent setting is indicated by a second heading placed at an angle so that positive $y$ of the initial group is to the right when the heading is horizontal. Such inequivalent settings occur for 6 of the 37 minimum steps of stress-induced symmetry lowering: $4 / \mathrm{mmm}$ to $\mathrm{mmm}$, $\mathrm{mmm}$ to $2 / \mathrm{m}$, $4 \mathrm{~mm}$ to $\mathrm{mm} 2,422$ to $222, \mathrm{~mm} 2$ to $\mathrm{m}$, and 222 to 2 . In the case of $4 / \mathrm{mmm}$ to $\mathrm{mmm}$ the two headings are written as $4 / \mathrm{mmm} \rightarrow \stackrel{\downarrow}{\mathrm{n} m} \mathrm{\downarrow} m$ to indicate that the
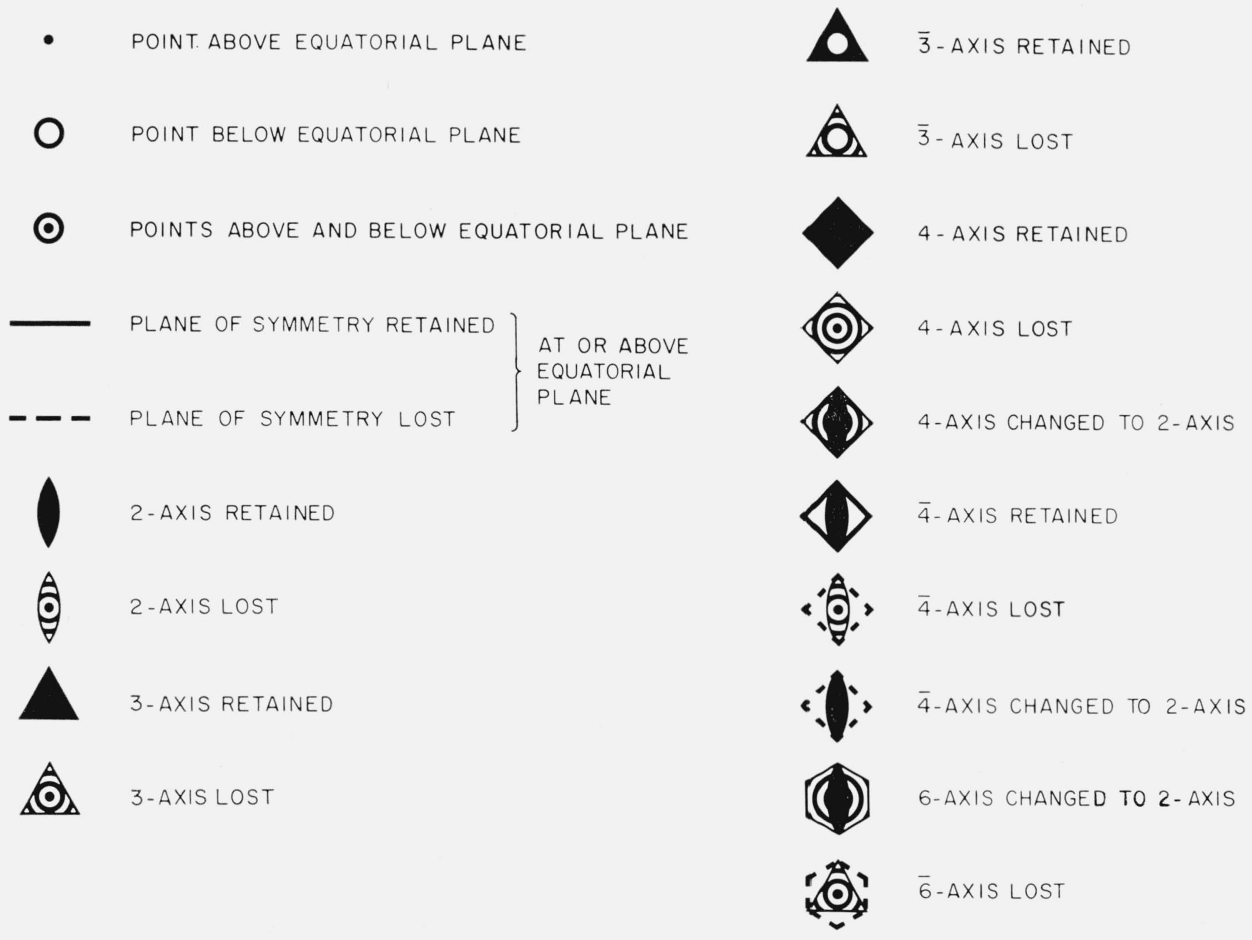

FigURE 1. 

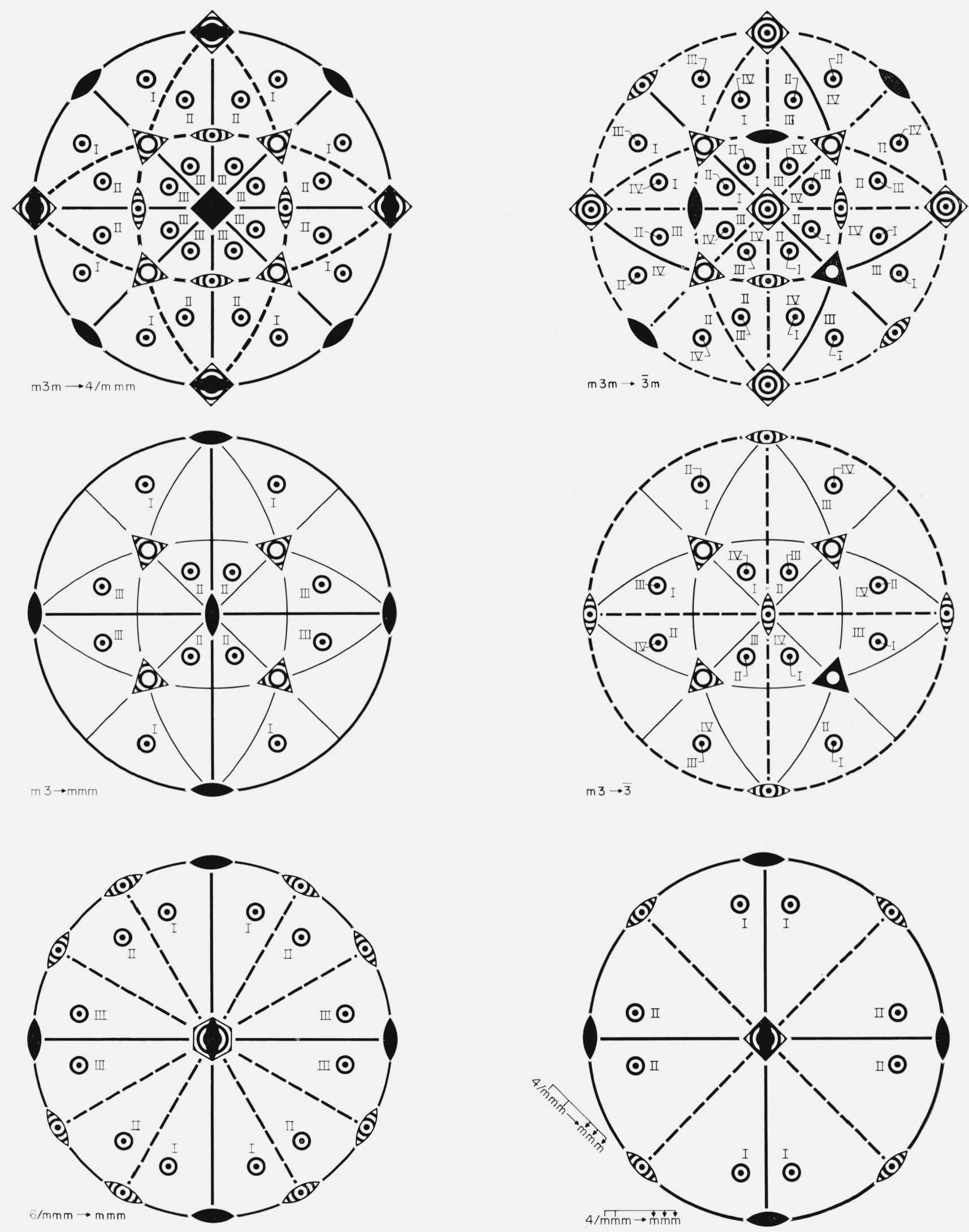

Figure 1-Continued 

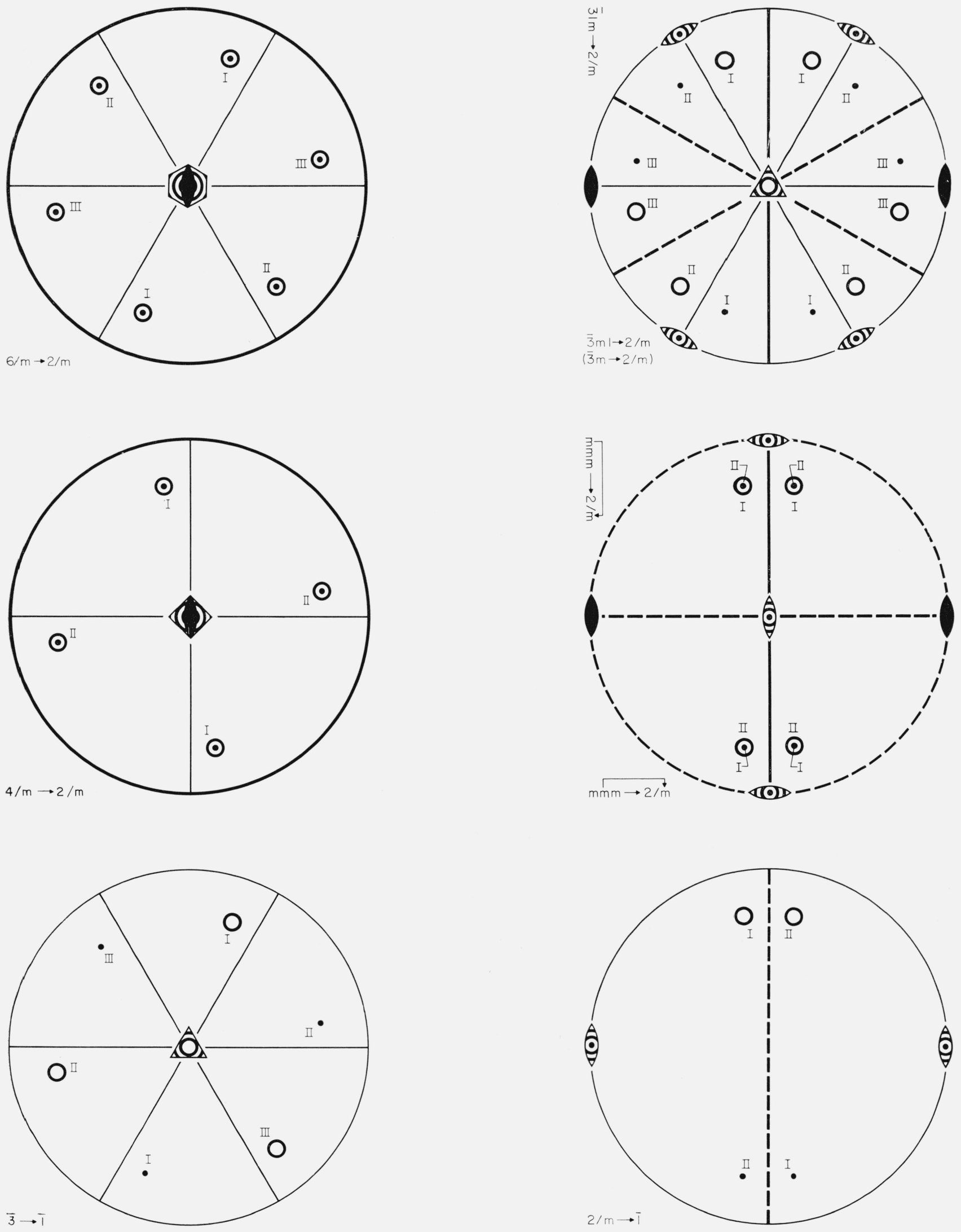

Figure 1-Continued 

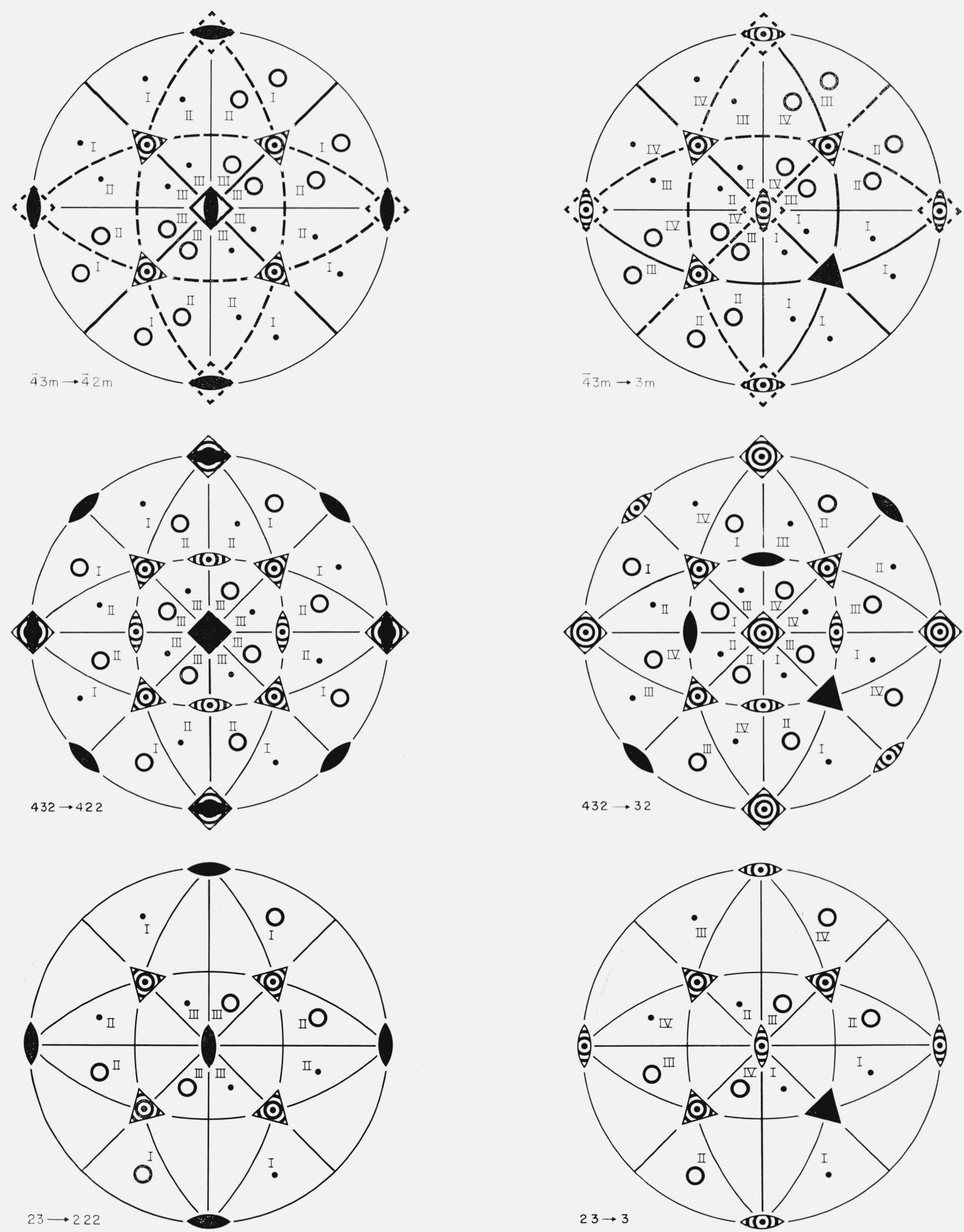

Figure 1-Continued 

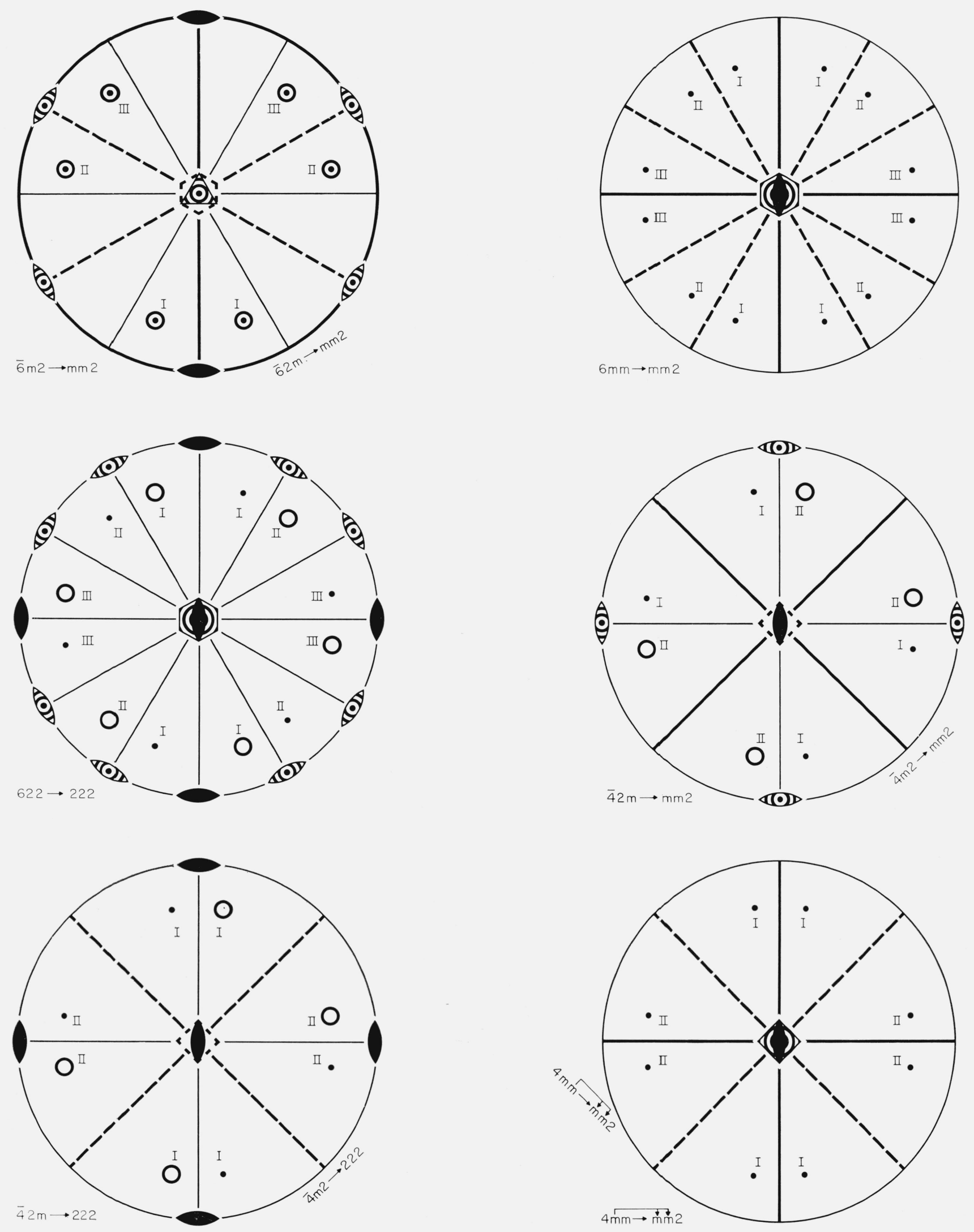

Figure 1-Continued 

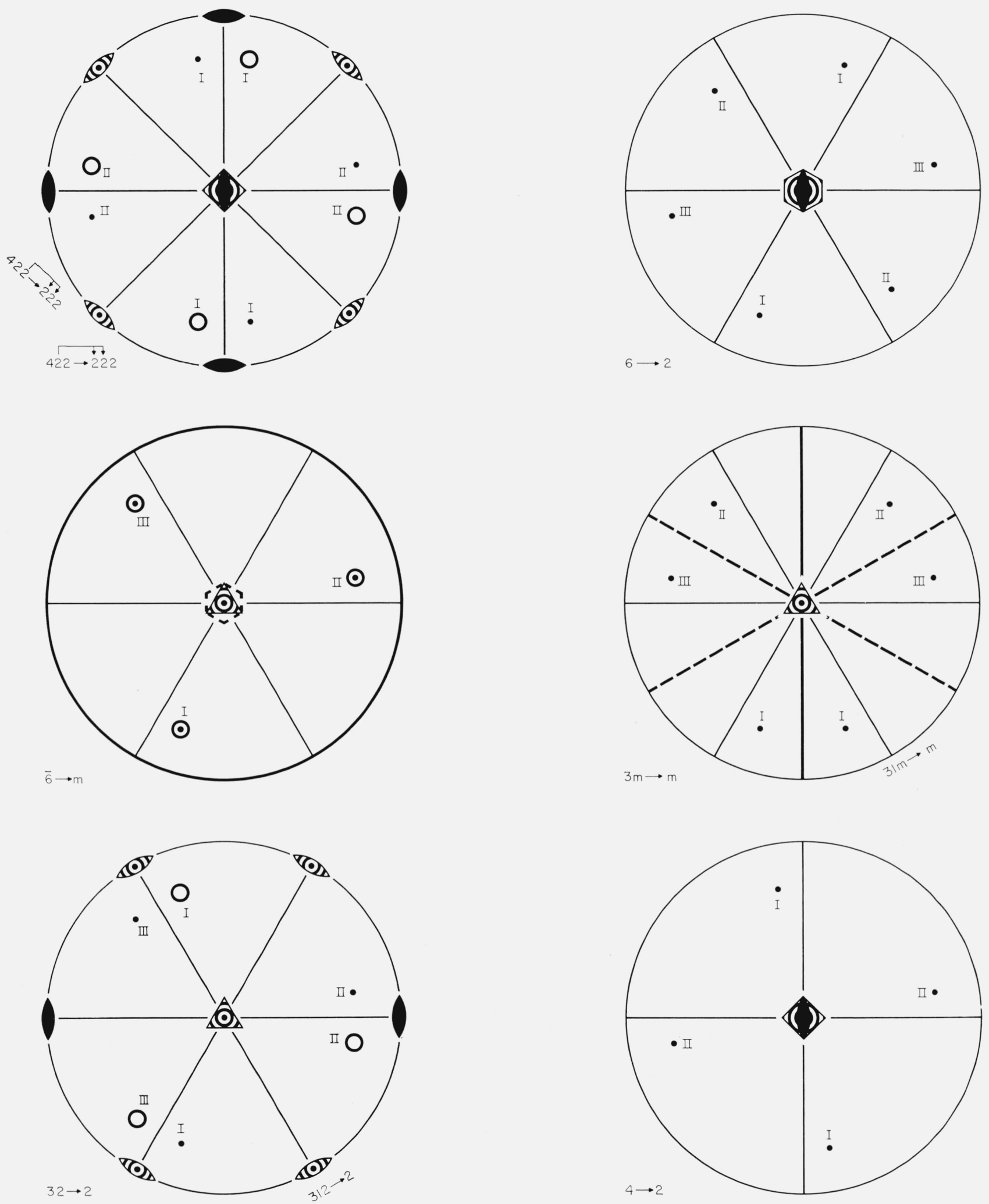

Figure 1-Continued 

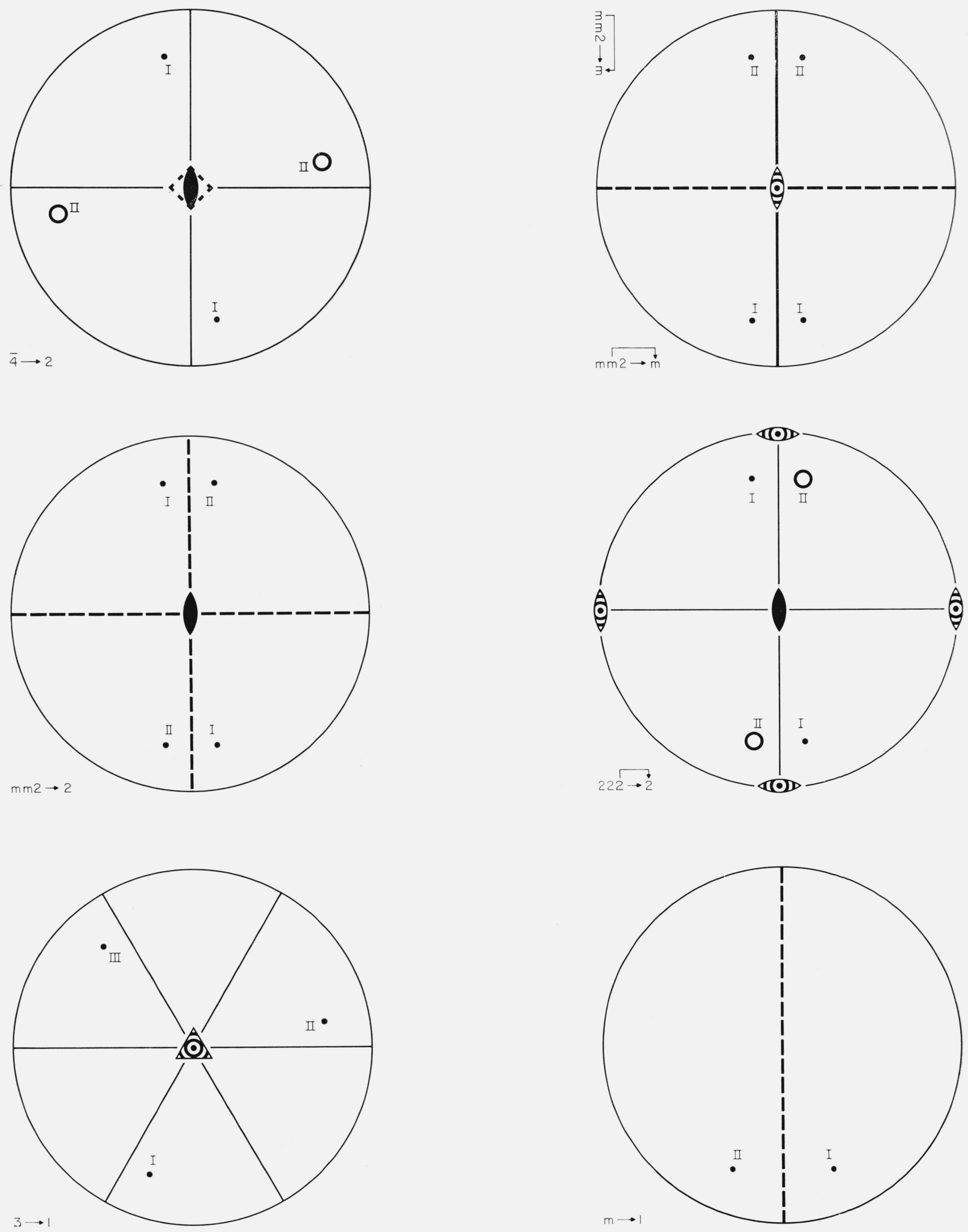

Figure 1-Continued 


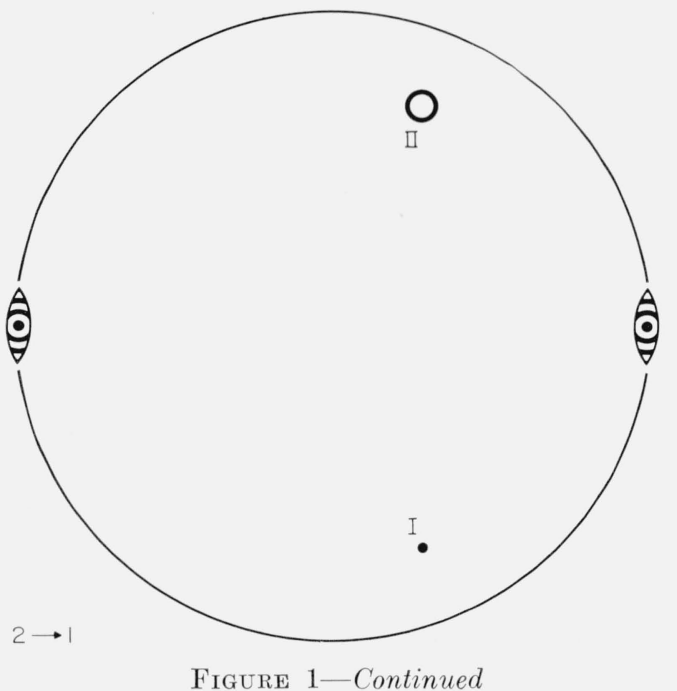

mirror planes perpendicular to $z, x$, and $y$ are preserved and as $4 / \mathrm{mmm} \rightarrow \stackrel{\mathrm{m} m}{\mathrm{~m}}$ to indicate that the mirror planes perpendicular to $z,[1 \overline{1} 0]$, and [110] are preserved. Similar headings are given for the other cases but this scheme does not permit three labels for the three inequivalent ways for $\mathrm{mmm}$ to $2 / \mathrm{m}$ and 222 to 2 .

Two headings are given on some of the other stereograms even though the subgroup does not occur in inequivalent ways. This is done for $\overline{3} \mathrm{~m}$ to $2 / \mathrm{m}$, $\overline{6} \mathrm{~m} 2$ to $\mathrm{mm} 2, \overline{4} 2 \mathrm{~m}$ to $\mathrm{mm} 2, \overline{4} 2 \mathrm{~m}$ to 222,32 to 2 , and $3 \mathrm{~m}$ to $\mathrm{m}$ to draw attention to the fact that an alternative setting is used sometimes for diverse reasons such as in describing crystals which in the morphologically conventional setting would have cells with a larger than minimum number of lattice points per cell.

A general direction (one not carried into itself by any of the operations of a point group under consideration) will give rise to a set of $n\left(\mathrm{PG}_{0}\right)$ equivalent directions where $n\left(\mathrm{PG}_{0}\right)$ is the order of the initial point group $\mathrm{PG}_{0}$. This set splits into $\frac{n\left(\mathrm{PG}_{0}\right)}{n\left(\mathrm{PG}_{1}\right)}$ subsets of $n\left(\mathrm{PG}_{1}\right)$ directions each, when the original point group is lowered to a subgroup $\mathrm{PG}_{1}$ of order $n\left(\mathrm{PG}_{1}\right)$. Each stereogram shows a set of $n\left(\mathrm{PG}_{0}\right)$ general directions for the initial point group; directions intersecting the reference sphere (from which the stereogram is derived) on the upper hemisphere (projector through the south pole) are represented by points and those intersecting the lower hemisphere (projector through the north pole) by circles. The directions which belong to the same subset under the operations of the subgroup for the stereogram are given the same Roman number.

When directions are shown in pairs (upper and lower hemispheres) one Roman numeral indicates that those two directions belong to the same subset. Otherwise, two separate Roman numerals are given; the one on the upper hemisphere has a connecting line to the center.

\section{Description of Stress Tables}

The symmetry elements of uniaxial stress are those of an ellipsoid of revolution [3,5]; i.e., an $\infty$-fold axis perpendicular to a mirror plane with every plane containing the $\infty$-fold axis also being a mirror plane. Tables 1 and 2 list the orientation of the $\infty$-fold axis suitable for each of the minimum steps of symmetry lowering. Each of the entries in the tables can be checked by superposing the above named symmetry elements on the stereogram of the initial point group with the $\infty$-fold axis oriented as specified. The tables also list appropriate orientations for all but three of the compound steps of symmetry lowering: $\mathrm{m} 3 \mathrm{~m}$ to $\mathrm{mmm}$ in one setting, $\overline{4} 3 \mathrm{~m}$ to 222 , and 432 to 222 in one setting; none of these three can be accomplished by uniaxial stress. For example, consider the reduction of $\mathrm{m} 3 \mathrm{~m}$ to $\mathrm{mmm}$ with the mirror planes of the latter perpendicular to the $x, y$, and $z$ axes of the former. To retain a mirror plane under uniaxial stress, the axis of the stress must be either parallel or perpendicular to the plane. For the present setting of $\mathrm{mmm}$ this requires the stress axis parallel to $x, y$, or $z$; these orientations result in the preservation of a four-fold axis and the reduction is to $4 / \mathrm{mmm}$ rather than $\mathrm{mmm}$. The other setting of $\mathrm{mmm}$ as a subgroup of $\mathrm{m} 3 \mathrm{~m}$ can, however, be reached by tensile stress directed along [110].

\section{Discussion}

\subsection{Splitting of a General Position in a Space Group Into Subsets of Sites}

There is a close correspondence between equivalence of directions under point-group operations and equivalence of sites in a crystal associated with one lattice point under space-group operations. There are as many equivalent general sites per lattice point of a crystal as there are equivalent general directions for the point group of the crystal. The symmetry operation connecting two sites associated with the same lattice point of a crystal must be the same as far as general rotations (i.e., proper rotation, reflection, or inversion) are concerned as the point-group operation connecting the isomorphic directions for the point group. A space-group operation in addition to a general rotation may also produce a translation which is a simple integral fraction of a lattice vector. In 73 of the space groups, termed the symmorphic space groups [12], the full point group is contained in the space group and a set of general positions associated with one lattice point must lie on a sphere surrounding that lattice point. The points representing directions in the stereograms of the present paper may be regarded as points on the surface of the reference sphere of the stereogram and so directly represent a set of general sites for a symmorphic space group [13] with the same point group. The symmorphic space groups, associated with each point group are listed in the last columns of tables 3 and 4 . For a nonsymmorphic space group one can begin with a general site and produce a set of equivalent 
TABLE 1. General orientation of homogeneous tensile stress (or biaxial stress if required) causing symmetry reduction

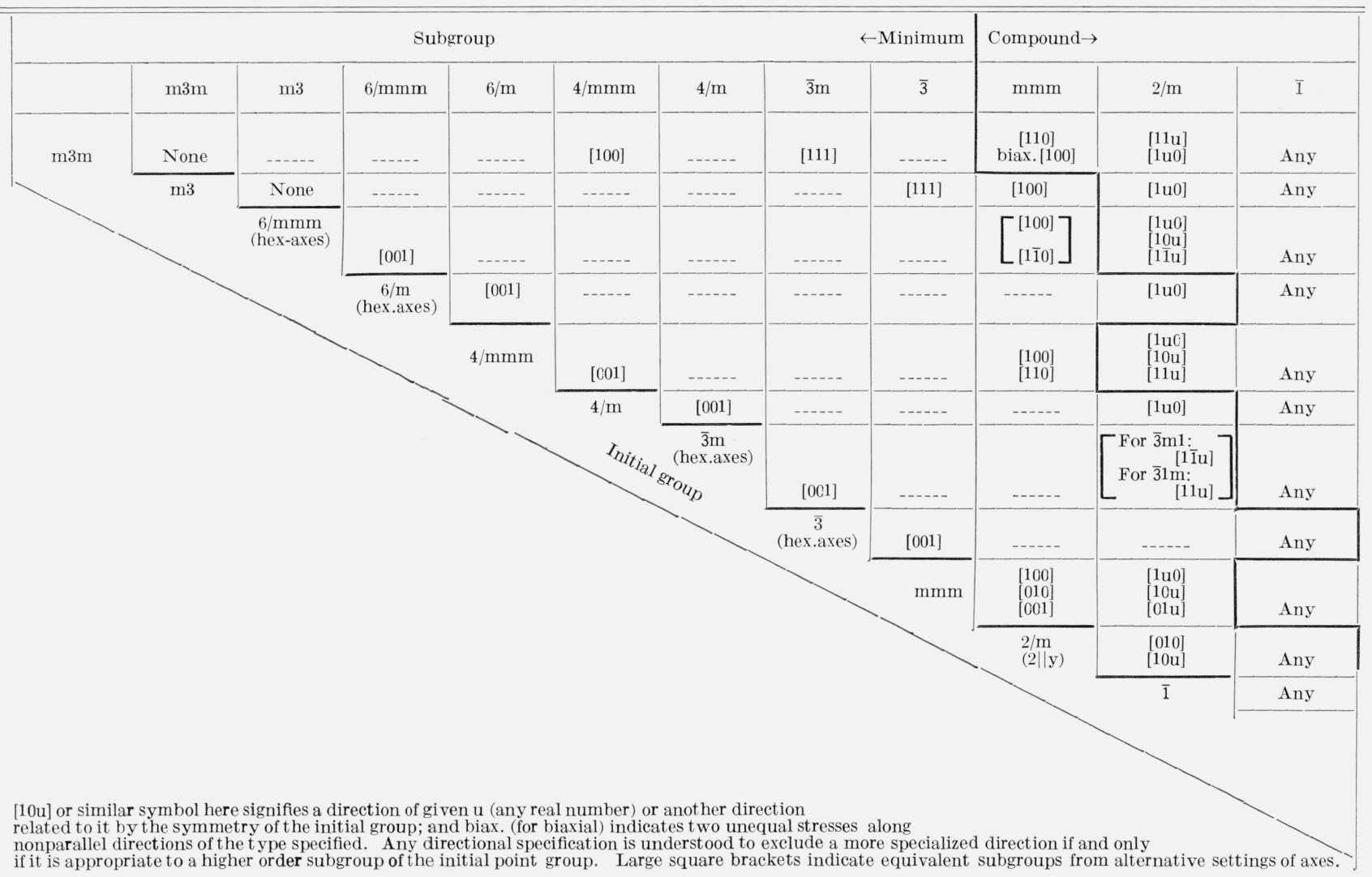

sites by using first the purely rotational operations and then the operations having fractional translation also. The former produce points on a sphere and the latter may be looked upon as first rotating to produce a point also on the sphere and then translating the point off the sphere. The points on the stereograms of the present paper can still be used as a representation of a set of general sites if it is recognized that the stereogram describes only the rotational part of the symmetry operation and that for a nonsymmorphic space group some of the points would be displaced from the surface of the reference sphere by translation of an integral fraction of a lattice vector. The pattern of splitting of a general position into subsets under stress is, however, the same for all space groups, symmorphic or nonsymmorphic. That pattern is associated with a given point-group-to-point-group reduction because the operations in the space group which are lost under stress are always isomorphic to the same operations in the point group.

All of the possible space-group-to-space-group reductions associated with each of the point-group-topoint-group transitions induced by stress have been tabulated [10]. For any symmorphic space-groupto-space-group reduction in this tabulation one can collect the coordinates of a set of equivalent general sites into subsets using the present stereograms to determine the permutations and changes of sign of $x, y$, and $z$ coordinates which produce equivalent sites in the subgroup. The same permutations and sign changes must also hold for any nonsymmorphic space group associated with the same point-group-to-pointgroup reduction; any additional translation can then be taken from the International Tables [11] because the permutations and sign of the $x y z$ coordinates uniquely identify each site of a set of equivalent sites as given there. This has been done for the general position in each of the 230 space groups, and tables of the splitting of a set of equivalent general sites into subsets are available $[6,14]$ for all minimum steps of symmetry lowering.

\subsection{Splitting of a Special Postion in a Space Group Into Subsets of Sites}

One can specialize a direction in a point group so that it is carried into itself by some of the operations of the point group other than the identity. All such operations together with the identity must form a group; let $\mathrm{PGS}_{0}$ represent this group and $n\left(\mathrm{PGS}_{0}\right)$ represent its order. Then there will be $n\left(\mathrm{PG}_{0}\right)$ / $n\left(\mathrm{PGS}_{0}\right)$ distinct directions making up a set of equivalent specialized directions of this type. The process of specialization can be visualized by picturing the motion of one of the points on any of the stereograms of figure 1. All of the other points on the same stereogram must move simultaneously to remain symmetry related. Specialization requires movement to make two or more points coincide so that the specialized direction is invariant under the opera- 
TABLE 2. General orientation of homogeneous tensile stress (or biaxial stress if required) causing symmetry reduction. (Symbols and notation as for table 1.)

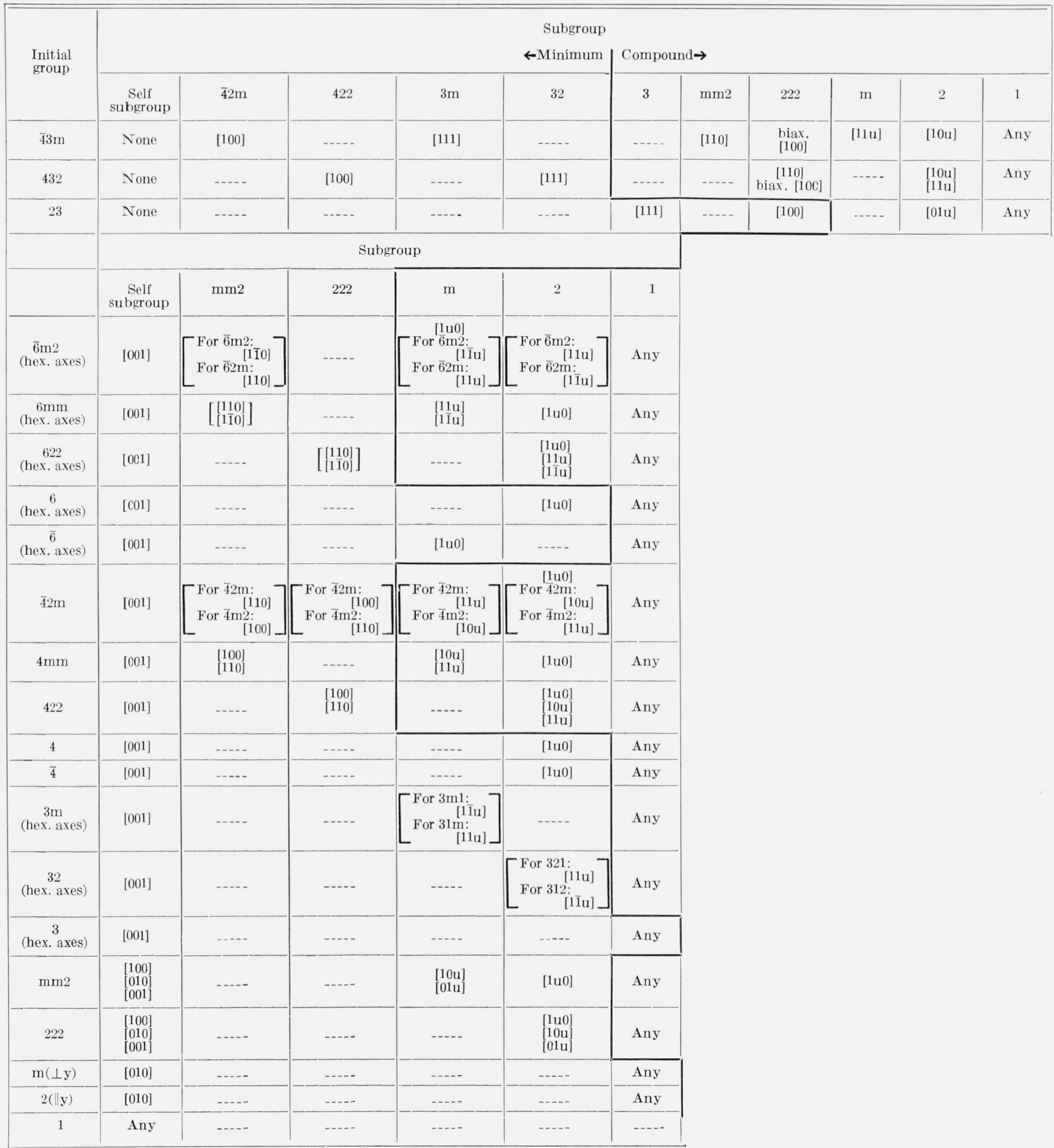

tions which related the now coincident directions. Several types of behavior occur separately or at the same time (in exact correspondence to the treatment of special positions in space groups [10]):

(1) Two points belonging to different subsets may be brought into coincidence on svmmetry lost so that the number of sets into which the set of specialized direction splits under stress is fewer than the number of subsets resulting from the set of general directions. For example, in the stereogram for 432 to 422 the motion of a point labeled I nearest [111] onto the threefold axis along [111] causes points I, II, and III to coincide. Thus, in the unstressed crystal of point group 432 a direction of symmetry $\mathrm{PGS}_{0}=3$ gives 
rise to a total of $n(432) / n(3)=24 / 3=8$ equivalent directions. In the stressed crystal the direction [111] has $\mathrm{PGS}_{1}=1$ so $n\left(\mathrm{PG}_{1}\right) / n\left(\mathrm{PGS}_{1}\right)=n(422) / n(1)=8 / 1$ $=8$ and the specialization has changed a set of 24 splitting into 3 subsets of 8 each into a set of 8 that does not split under this stress. Unequal splitting can be demonstrated, for example, for $3 \mathrm{~m}$ to $2 / \mathrm{m}$, as mentioned in the introduction. Let the points labeled II and III be brought into coincidence by moving them onto the dashed lines representing lost mirror planes: this requires the points labeled I to move onto the mirror plane which is retained. In the unstressed crystal all directions have $\mathrm{PGS}_{0}=\mathrm{m}$ with $n(\overline{3} \mathrm{~m}) / n(\mathrm{~m})=12 / 2=6$. In the stressed crystal one subset has $\mathrm{PGS}_{1}{ }^{(1)}=\mathrm{m}$ with a number in the set of $n(2 / \mathrm{m}) / n(\mathrm{~m})=4 / 2=2$; the other subset has $\mathrm{PGS}_{1}{ }^{(2)}=1$ and 4 directions in the set.

(2) Directions in the same subset may become identical in clusters of two or more each on symmetry retained. The specialized directions in this subset are necessarily specialized with respect to the reduced point group. In the above example of $\overline{3} \mathrm{~m}$ to $2 / \mathrm{m}$ the coincident points labeled I were still specialized with respect to $2 / \mathrm{m}$.

(3) The number of degrees of freedom in a subset may exceed the number in the original set of special directions because of loss of a constraint resulting from loss of symmetry. In the above example of $\overline{3} \mathrm{~m}$ to $2 / \mathrm{m}$ the points of subsets resulting from coincidence of II and III were specialized to lie on mirror planes of $\overline{3} \mathrm{~m}$ and so had only one degree of freedom; these planes are lost in going to $2 / \mathrm{m}$ as shown in the stereogram so that the points have two degrees of freedom with respect to $2 / \mathrm{m}$.
The fourth columns of tables 3 and 4 list the possible specializations to $\mathrm{PGS}_{0}$ which can be accomplished by moving the general direction on the stereogram for each point group. Sites having position point symmetry, $\mathrm{PPS}_{0}$, equal to each possible $\mathrm{PGS}_{0}$ must occur in each of the symmorphic space groups. The latter are listed in the last columns of tables 3 and 4 . There are, however, sites of different point symmetry which can occur even in some of the symmorphic point groups because of symmetry operations which are interwoven between the lattice points when the latter are chosen to have the full point symmetry. Thus, in Fmm2 sites of symmetry 2 occur halfway between lattice points but the general direction of the stereogram for $\mathrm{mm} 2$ can not be specialized just to 2 but becomes mm2. Many such cases occur; columns 5-7 of tables 3 and 4 list all of the additional position point symmetries (which must be a subgroup of the initial point group) occurring in the symmorphic or nonsymmorphic space groups associated with each point group and not already listed in columns 4 . One might suppose that all of the subgroups of $\mathrm{PG}_{0}$ would occur as position point symmetries in some space group associated with $\mathrm{PG}_{0}$, but column 8 of table 3 shows that a few exceptions exist.

The behavior of a special position of symmetry $\mathrm{PPS}_{0}$ which is not a possible $\mathrm{PGS}_{0}$ of the point group can be visualized by using two or more of the stereograms together. Under a stress which causes a minimum step of symmetry lowering of $\mathrm{PG}_{0}$ to $\mathrm{PG}_{1}$ the group $\mathrm{PPS}_{0}$ must either remain unchanged or undergo a step of symmetry lowering but the latter need not be a minimum step. For example, $\mathrm{PPS}_{0}=$

TABLE 3. Point symmetries reached by specializing general directions for each centrosymmetric point group as applicable to stereograms; point symmetries occurring in symmorphic and nonsymmorphic space groups but not on stereograms; and point symmetries which, while forming a subgroup of the initial point group, are not found in any associated space group

\begin{tabular}{|c|c|c|c|c|c|c|c|c|}
\hline \multirow{3}{*}{ Int. tables ${ }^{11}$} & \multirow{3}{*}{$\begin{array}{l}\text { Initial group } \\
\text { Schoenflies }{ }^{11}\end{array}$} & \multirow{3}{*}{$\operatorname{Shubni-}_{\text {kov }}^{16}$} & \multicolumn{5}{|c|}{ Subgroups (other than 1) } & \multirow{3}{*}{$\begin{array}{l}24 \text { centrosymmetric space- } \\
\text { groups with full point } \\
\text { symmetry of initial point } \\
\text { group }\end{array}$} \\
\hline & & & \multirow{2}{*}{$\begin{array}{l}\text { occurring on the } \\
\text { stereogram and in } \\
\text { at least one space } \\
\text { group associated } \\
\text { with the initial } \\
\text { point group }\end{array}$} & \multicolumn{3}{|c|}{ not occurring on stereogram but in at least one } & \multirow{2}{*}{$\begin{array}{l}\text { not on ster- } \\
\text { eogram nor } \\
\text { in any asso- } \\
\text { ciated space } \\
\text { group }\end{array}$} & \\
\hline & & & & $\begin{array}{l}\text { symmorphic, but } \\
\text { not in associated } \\
\text { nonsymmorphic } \\
\text { space groups }\end{array}$ & $\begin{array}{l}\text { symmorphic and } \\
\text { at least one associ- } \\
\text { ated nonsymmor- } \\
\text { phic space groups }\end{array}$ & $\begin{array}{l}\text { nonsymmorphic } \\
\text { but not in associ- } \\
\text { ated symmorphic } \\
\text { space groups }\end{array}$ & & \\
\hline $\mathrm{m} 3 \mathrm{~m}$ & $\mathrm{O}_{\mathrm{h}}$ & $\overline{6} / 4$ & $\underset{\mathrm{m}}{4 \mathrm{~mm}}, 3 \mathrm{~m}, \mathrm{~mm} 2$ & $\mathrm{~m} 3 \mathrm{~m}, 4 / \mathrm{mmm}$ & $\begin{array}{l}\overline{4} 3 \mathrm{~m}, \overline{3} \mathrm{~m}, \overline{4} 2 \mathrm{~m} \\
\mathrm{~mm} 2,2\end{array}$ & $\begin{array}{l}\mathrm{m} 3,432,23,4 / \mathrm{m} \\
\quad 422, \overline{3}, 32,4, \overline{4} \\
\quad 222,3\end{array}$ & $2 / \mathrm{m}, \overline{1}$ & $\mathrm{Pm} 3 \mathrm{~m}, \mathrm{Fm} 3 \mathrm{~m}, \mathrm{Im} 3 \mathrm{~m}$ \\
\hline $\mathrm{m} 3$ & $\mathrm{~T}_{\mathrm{h}}$ & $\overline{6} / 2$ & $\mathrm{~mm} 2,3, \mathrm{~m}$ & $\mathrm{~m} 3, \mathrm{mmm}, 2 / \mathrm{m}$ & $23, \overline{3}$ & 222,2 & $\overline{1}$ & $\operatorname{Pm} 3, \mathrm{Fm} 3, \operatorname{Im} 3$ \\
\hline $6 / \mathrm{mmm}$ & $\mathrm{D}_{6 \mathrm{~h}}$ & $\mathrm{~m} \cdot 6: \mathrm{m}$ & $6 \mathrm{~mm}, \mathrm{~mm} 2, \mathrm{~m}$ & $6 / \mathrm{mmm}, \mathrm{mmm}$ & $\overline{6} \mathrm{~m} 2,3 \mathrm{~m}$ & $\begin{array}{l}\text { 6/m, } 622, \overline{3} \mathrm{~m}, 32 \\
\quad 6, \overline{6}, 222,2 / \mathrm{m} \\
\quad 3,2\end{array}$ & $\overline{3}, \overline{1}$ & $\mathrm{P} 6 / \mathrm{mmm}$ \\
\hline $4 / \mathrm{mmm}$ & $\mathrm{D}_{4 \mathrm{~h}}$ & $\mathrm{~m} \cdot 4: \mathrm{m}$ & $4 \mathrm{~mm}, \mathrm{~mm} 2, \mathrm{~m}$ & $4 / \mathrm{mmm}$ & $\underset{2}{\overline{4} 2 \mathrm{~m}}, \mathrm{mmm}, 2 / \mathrm{m}$ & $\begin{array}{l}4 / \mathrm{m}, 422,4, \overline{4} \\
222, \overline{1}\end{array}$ & & $\mathrm{P} 4 / \mathrm{mmm}, \mathrm{I} 4 / \mathrm{mmm}$ \\
\hline $6 / \mathrm{m}$ & $\mathrm{C}_{6 \mathrm{~h}}$ & $6: \mathrm{m}$ & $6, \mathrm{~m}$ & $6 / \mathrm{m}, 2 / \mathrm{m}, 2$ & $\overline{6}, 3$ & $\overline{3}, \overline{1}$ & $-\cdots$ & $\mathrm{P} 6 / \mathrm{m}$ \\
\hline$\overline{3} \mathrm{~m}$ & $\mathrm{D}_{3 \mathrm{~d}}$ & $\overline{6} \cdot \mathrm{m}$ & $3 \mathrm{~m}, \mathrm{~m}, 2$ & $\overline{3} \mathrm{~m}, 2 / \mathrm{m}$ & 32,3 & $\overline{3}, \overline{1}$ & $\ldots$ & $\mathrm{P} \overline{3} 1 \mathrm{~m}, \mathrm{P} \overline{3} \mathrm{~m} 1, \mathrm{R} \overline{3} \mathrm{~m}$ \\
\hline $4 / \mathrm{m}$ & $\mathrm{C}_{4 \mathrm{~h}}$ & $4: \mathrm{m}$ & $4, \mathrm{~m}$ & $4 / \mathrm{m}$ & $\overline{4}, 2 / \mathrm{m}, 2, \overline{1}$ & $\begin{array}{ll} \\
\end{array}$ & & $\mathrm{P} 4 / \mathrm{m}, 14 / \mathrm{m}$ \\
\hline $\mathrm{mmm}$ & $\mathrm{D}_{2 \mathrm{~h}}\left(\mathrm{~V}_{\mathrm{h}}\right)$ & $\mathrm{m} \cdot 2: \mathrm{m}$ & $\mathrm{mm} 2, \mathrm{~m}$ & $\mathrm{mmm}$ & $222,2 / \mathrm{m}, 2, \overline{1}$ & & & $\begin{array}{l}\text { Pmmm, Cmmm, Immm, } \\
\text { Fmmm }\end{array}$ \\
\hline$\overline{3}$ & $\mathrm{C}_{3 \mathrm{i}}\left(\mathrm{S}_{6}\right)$ & $\overline{6}$ & 3 & $\overline{3}, \overline{1}$ & - & $\therefore$ & - & $\mathrm{P} \overline{3}, \mathrm{R} \overline{3}$ \\
\hline $2 / \mathrm{m}$ & $\mathrm{C}_{2 \mathrm{~h}}$ & $2: \mathrm{m}$ & $\mathrm{m}, 2$ & $2 / \mathrm{m}$ & $\overline{1}$ & $-\cdots$ & - & $\mathrm{P} 2 / \mathrm{m}, \mathrm{C} 2 / \mathrm{m}$ \\
\hline$\overline{1}$ & $\mathrm{C}_{\mathrm{i}}\left(\mathrm{S}_{2}\right)$ & $\overline{2}$ & & $\overline{1}$ & & & & $\mathrm{P} \overline{1}$ \\
\hline
\end{tabular}


TABLE 4. Point symmetries reached by specializing general directions for each noncentrosymmetric point group as applicable to stereograms; and point symmetries occurring in symmorphic and nonsymmorphic space groups but not on stereograms

(Point symmetries forming a subgroup of any initial noncentrosymmetric point group are found in at least one associated space group.)

\begin{tabular}{|c|c|c|c|c|c|c|c|}
\hline \multirow{3}{*}{$\begin{array}{l}\text { Int. } \\
\text { tables } 11\end{array}$} & \multirow{3}{*}{$\begin{array}{l}\text { Initial group } \\
\text { Schoen- } \\
\text { flies }^{11}\end{array}$} & \multirow{3}{*}{$\begin{array}{l}\text { Shubni- } \\
\text { kov } 16\end{array}$} & \multicolumn{4}{|c|}{ Subgroups (other than 1) } & \multirow{3}{*}{$\begin{array}{l}49 \text { noncentrosymmetric } \\
\text { space groups with full } \\
\text { point symmetry of ini- } \\
\text { tial point group }\end{array}$} \\
\hline & & & \multirow{2}{*}{$\begin{array}{l}\text { occurring on the ster- } \\
\text { eogram and in at } \\
\text { least one space group } \\
\text { associated with the } \\
\text { initial point group }\end{array}$} & \multicolumn{3}{|c|}{ not occurring on stereogram but in at least one } & \\
\hline & & & & $\begin{array}{l}\text { symmorphic but not } \\
\text { in associated non- } \\
\text { symmorphic space } \\
\text { groups }\end{array}$ & $\begin{array}{l}\text { symmorphic and at } \\
\text { least one associated } \\
\text { nons ymmorphic } \\
\text { space groups }\end{array}$ & $\begin{array}{l}\text { nonsymmorphic but } \\
\text { not in associated } \\
\text { symmorphic space } \\
\text { groups }\end{array}$ & \\
\hline $43 \mathrm{~m}$ & $\mathrm{~T}_{\mathrm{d}}$ & $3 / \overline{4}$ & $3 \mathrm{~m}, \mathrm{~mm} 2, \mathrm{~m}$ & $43 \mathrm{~m}, 42 \mathrm{~m}$ & 4,2 & $23,222,3$ & $\mathrm{P} \overline{4} 3 \mathrm{~m}, \mathrm{~F} \overline{4} 3 \mathrm{~m}, \mathrm{I} \overline{4} 3 \mathrm{~m}$ \\
\hline 432 & $\mathrm{O}$ & $3 / 4$ & $4,3,2$ & 432,422 & $23,32,222$ & - & P432, F432, I432 \\
\hline 23 & $\mathrm{~T}$ & $3 / 2$ & 3,2 & 23,222 & 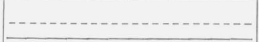 & (n) & $\mathrm{P} 23, \mathrm{~F} 23, \mathrm{I} 23$ \\
\hline $6 \bar{m} 2$ & $\mathrm{D}_{3 \mathrm{~h}}$ & $\mathrm{~m} \cdot 3: \mathrm{m}$ & $3 \mathrm{~m}, \mathrm{~mm} 2, \mathrm{~m}$ & $6 \mathrm{~m} 2$ & 6,3 & 32,2 & $\mathrm{P} \overline{\operatorname{c}} \mathrm{m} 2, \mathrm{P} \overline{6} 2 \mathrm{~m}$ \\
\hline $6 \mathrm{~mm}$ & $\mathrm{C}_{6 \mathrm{v}}$ & $6 \cdot \mathrm{m}$ & $6 \mathrm{~mm}, \mathrm{~m}$ & $\mathrm{~mm} 2$ & $3 \mathrm{~m}$ & $6,3,2$ & $\mathrm{P} 6 \mathrm{~mm}$ \\
\hline 622 & $\mathrm{D}_{3}$ & $6: 2$ & 6,2 & 622 & $32,222,3$ & - & $\mathrm{P} 622$ \\
\hline$\overline{4} 2 \mathrm{~m}$ & $\mathrm{D}_{2 \mathrm{~d}}\left(\mathrm{~V}_{\mathrm{d}}\right)$ & $\overline{4} \cdot \mathrm{m}$ & $\mathrm{mm} 2, \mathrm{~m}, 2$ & $42 \mathrm{~mm}$ & 4,222 & --- & $\begin{array}{r}\mathrm{P} \overline{4} 2 \mathrm{~m}, \mathrm{P} \overline{4} \mathrm{~m} 2, \\
\mathrm{I} \overline{4} \mathrm{~m} 2 \mathrm{~m} 2,\end{array}$ \\
\hline $4 \mathrm{~mm}$ & $\mathrm{C}_{4 \mathrm{v}}$ & $4 \cdot \mathrm{m}$ & $4 \mathrm{~mm}, \mathrm{~m}$ & - & $\mathrm{mm} 2$ & 4,2 & $\mathrm{P} 4 \mathrm{~mm}, \mathrm{I} 4 \mathrm{~mm}$ \\
\hline 422 & $\mathrm{D}_{4}$ & $4: 2$ & 4,2 & 422 & 222 & - & P422, I422 \\
\hline 6 & $\mathrm{C}_{6}$ & 6 & 6 & - & 3,2 & - & P6 \\
\hline$\overline{6}$ & $\mathrm{C}_{3 \mathrm{~h}}$ & $3: \mathrm{m}$ & $3, \mathrm{~m}$ & 6 & - & - & $\mathrm{P} \overline{6}$ \\
\hline $3 \mathrm{~m}$ & $\mathrm{C}_{3 \mathrm{v}}$ & $3 \cdot \mathrm{m}$ & $3 \mathrm{~m}, \mathrm{~m}$ & - & 3 & - & P3m1, P31m, R3m \\
\hline 32 & $\mathrm{D}_{3}$ & $3: 2$ & 3,2 & 32 & - & - & P312, P321, R32 \\
\hline 4 & $\mathrm{C}_{4}$ & 4 & 4 & -1-1, & 2 & - & $\mathrm{P} 4, \mathrm{I} 4$ \\
\hline$\overline{4}$ & $\mathrm{~S}_{4}$ & $\overline{4}$ & 2 & 4 & - & (- & $\mathrm{P} \overline{4}, \mathrm{I} \overline{4}$ \\
\hline $\mathrm{mm} 2$ & $\mathrm{C}_{2 \mathrm{v}}$ & $2 \cdot \mathrm{m}$ & $\mathrm{mm} 2, \mathrm{~m}$ & 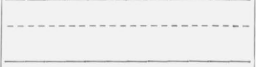 & 2 & & $\begin{array}{l}\text { Fmm2, Imm2, } \\
\text { Pmm2, Cmm2, Amm2 }\end{array}$ \\
\hline 222 & $\mathrm{D}_{2}(\mathrm{~V})$ & $2: 2$ & 2 & 222 & & & $\begin{array}{r}\mathrm{P} 222, \mathrm{C} 222, \mathrm{I} 222, \\
\mathrm{~F} 222 \\
\end{array}$ \\
\hline 3 & $\mathrm{C}_{3}$ & 3 & 3 & - & ( & - & P3, R3 \\
\hline $\mathrm{m}$ & $\mathrm{C}_{\mathrm{s}}\left(\mathrm{C}_{1 \mathrm{~h}}\right)$ & $1 \cdot \mathrm{m}$ & $\mathrm{m}$ & - & ( & - & $\mathrm{Pm}, \mathrm{Cm}$ \\
\hline 2 & $\mathrm{C}_{2}$ & 2 & 2 & - & - & - & $\mathrm{P} 2, \mathrm{C} 2$ \\
\hline 1 & $\mathrm{C}_{1}$ & 1 & 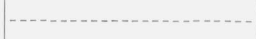 & $\ldots$ & & & P1 \\
\hline
\end{tabular}

$4 / \mathrm{m}$ occurs in some space groups associated with $\mathrm{PG}_{0}=\mathrm{m} 3 \mathrm{~m}$; tensile stress along [111] causes the minimum step $\mathrm{m} 3 \mathrm{~m}$ to $\overline{3} \mathrm{~m}$ but also causes the compound step in position point symmetry $4 / \mathrm{m}$ to $\overline{1}$. These results can be visualized from the stereograms for $\mathrm{m} 3 \mathrm{~m}$ to $\overline{3} \mathrm{~m}$ and $4 / \mathrm{m}$ to $2 / \mathrm{m}$. The latter shows the only minimum step for $4 / \mathrm{m}$ and shows that $\mathrm{m}$ perpendicular 4 must be preserved; the former shows that all mirror planes perpendicular to 4-axes are lost. The combination of a minimum step of symmetry lowering of $\mathrm{PG}_{0}$ with a compound step for $\mathrm{PGS}_{0}$ on a stereogram can also occur as the example of $\mathrm{m} 3 \mathrm{~m}$ to $\overline{3} \mathrm{~m}$ accompanied by $4 / \mathrm{mmm}$ to $\mathrm{m}$ shows.

This type of visualization can be applied to any $\mathrm{PPS}_{0}$ of $\mathrm{PG}_{0}$ but it should be noted that the orientation of $\mathrm{PPS}_{0}$ is fixed by $\mathrm{PG}_{0}$ so that a stereogram of $\mathrm{PPS}_{0}$ as initial group may require reorientation to correspond to the setting in $\mathrm{PG}_{0}$.

\subsection{Effect of Homogeneous Stress on Morphology of Crystals}

The identity of the 32 morphological crystal classes with the 32 three-dimensional point groups is well understood (Phillips [15]). A straight forward application therefore of the stereograms here presented would appear to be that concerned with changes in homogeneously strained crystals. From the practical experimental viewpoint, however, the application of homogeneous stress (other than isostatic) to a polyhedrally shaped object is difficult to visualize and more difficult to achieve. Apart from general mathematical treatment there seems so far to have been little interest by practical crystallographers in the homogeneous distortion of crystal shapes. For general forms minimum step degradations can be directly read from the figures as conversions to general forms of the subgroup. The number of now independent but co-existing forms in the subgroup is simply $\frac{n\left(\mathrm{PG}_{0}\right)}{n\left(\mathrm{PG}_{1}\right)}$ the ratio of the order of the initial to that of the final subgroup, the splitting factor sometimes called the index of the subgroup. It can simply be read off the stereograms given as the highest Roman figure designation of a direction on the stereogram of the parent group.

In this way for example a compressive stress 
II111] for a crystal belonging to $\mathrm{m} 3 \mathrm{~m}$ will convert one $\{h k l\}$ hex(akis)octahedron to four hexagonal bipyramids.

A randomly oriented stress will convert any general form into unrelated triclinic $\{h k l\}$ pedia (for noncentrosymmetric crystals) or $\{h k l\}$ pinacoids (for centrosymmetric crystals). The number of forms being simply the order (for noncentrosymmetric) or half the order (for centrosymmetric crystals) of the initial point group.

All possible intermediate compound steps can be read off tables 1 and 2. The forms produced from general forms in $\mathrm{PG}_{0}$ are always general forms $\{h k l\}$ in the ultimate subgroup and their number again equals the ratio of the order of the initial to that of the final point group.

When special forms are involved the procedure follows that outlined above for cell site co-ordinates; the only possible special forms being those listed in the fourth column of tables 3 and 4 .

Faces will belong to special forms in the subgroup if any one of the original symmetry operations, the stereographic representation of which is parallel to the pole of the face, is retained in the degradation.

When an initial point group has a number of equivalent symmetry operations, stress may lead to the loss of one or more of these operations, yet leave one or more of them in the subgroup. Directions specialized with respect to these initially equivalent symmetry operations will split unequally. For example, in the transformation $\mathrm{m} 3 \mathrm{~m}$ to $4 / \mathrm{mmm}$, a $\{100\}$ cube, with six faces, will split into a tetragonal basal pinacoid $\{001\}$ with two faces and a tetragonal (first order) prism $\{100\}$ with four faces.

No splitting of the special form will result if specialform poles can be regarded as having arisen from the superposition, on symmetry operations, of poles including at least one with each Roman-figure designation. The shape of the solid figure naturally will change. For example, as $\mathrm{m} 3 \mathrm{~m}$ transforms to $4 / \mathrm{mmm},\{111\}$ changes from a regular octahedron to a (2d order) tetragonal bypyramid. As $4 / \mathrm{mmm}$ transforms further to $\mathrm{mmm}$ in one orientation $\{111\}$ becomes a rhombic bipyramid, the general form of that class. In the $45^{\circ}$ position, however, it would split into two unrelated rhombic domes. Further symmetry degradation must also split the rhombic bipyramid, because it is the general form. In other words: the faces of the original octahedron \{111\} number eight, its faces cannot remain all equivalent when degradation proceeds to a subgroup of order less than eight.

In this way it is easy to deduce which crystallographic forms can be transformed by homogeneous stress into which others. Conversely, if there is no simple or compound subgroup relationship that will transform any one given form in any of the point groups in which it occurs into another form with an appropriate subgroup symmetry then there exists no homogeneous stress that can effect that transformation. For example as is well known to geometers - a tetrahedron, $\{111\}$ in $\overline{4} 3 \mathrm{~m}$, can be homogeneously deformed to a tetragonal sphenoid $\{111\}$ in $\overline{4} 2 \mathrm{~m}$, and to a rhombic bisphenoid, $\{111\}$ in 222 , but not to a rhombic dome, which also has four faces.

However, some care is necessary in deducing that a given transformation is impossible, because the special forms of some classes are indistinguishable from some special and, in one or two instances, even general forms (taken singly) of other classes.

There are a number of other applications of this kind of morphological discussion of homogeneously strained crystals. Temperature, pressure, or composition changes may lead to phase transformations (displacive) in which no primary bonds are broken in the solid [16]. Such changes may or may not be consistent with changes that could arise by homogeneous deformation as here described. This will introduce a further meaningful subclassification of the transformations discussed by Buerger [161.

Another, completely different, consideration may prove to be fruitful. Crystal growth results in shapes that often depart considerably from ideal symmetry of the atomic structure. A number of causes for these departures are established, and relatively well understood:-

1. Accidental asymmetry of supply of solute or melt to equivalent faces;

2. Retardation of growth of some faces by substrate interference or competition for solute or liquid with neighboring crystals;

3. Accidental differences in the small number of growth step sources on otherwise equivalent faces; and

4. Geometric complications arising from twinning.

An additional effect suggests itself. Stress during growth may make faces inequivalent causing unequal growth rate on otherwise equivalent faces. On examination of the crystals after stress removal the equivalence of the faces demanded by structural symmetry is reestablished, yet the asymmetry of shape persists.

This effect to be discerned may need to be studied deliberately and carefully under laboratory conditions. Certainly two outstanding anomalies (some diamond and cuprite crystals) of known minerals with morphology suggestive of lower symmetry than their established structure [17] can not be explained by homogeneous strain during growth. These two anomalies are accompanied by anomolous etch figures. Any stress induced inequivalence of structure, that gives rise only to homogeneous elastic strain. should vanish when the stress is removed.

\section{Appendix I}

We here present a simple, systematic, and exhaustive scheme for deriving all possible subgroups that can be reached by the action of homogeneous stress on any point group.

Let us introduce a number of concepts and symbols. First, consider any stress ellipsoid, $E_{p}$, which in shape and orientation is least restricted yet still consistent with any original point group (cf. table 13, reference 5). Stress-induced symmetry lowering can be caused only by an imposed stress of which the ellipsoid representation, $E_{s}$, does not conform to all the re- 
strictions in $E_{p}$. Point-group degradations for each initial point-group, $\mathrm{PG}_{0}$ can now be regarded as associated with liberalizations, $L$, of these restrictions imposed on $E_{p}$.

We will distinguish $L_{S}$ and $L_{0}$ liberalizing ellipsoid shape and orientation respectively. All PG degradations can be regarded as caused by one or both of these applied to $E_{p}$. We further classify into $L_{\mathrm{SR}}$, $L_{S T}, L_{01}$, and $L_{02}$.

$L_{\mathrm{SR}}$ is a shape liberalization leading to an ellipsoid of revolution with unique axis parallel to a crystal axis ${ }^{2}$ of order $>2$. As the only more restrictive stress ellipsoid is a sphere the operation $L_{\mathrm{SR}}$ need be considered only when $\mathrm{PG}_{0}$ is cubic, for which the $L_{\mathrm{SR}}$ operation produces one or two symmetry degradations depending on whether there are one or two nonequivalent, nonparallel axes of order $>2$ in $\mathrm{PG}_{0}$.

$L_{\mathrm{ST}}$ is a shape liberalization leading to a triaxial ellipsoid, the major axes of which are parallel to three mutually perpendicular crystal axes of even order, in $\mathrm{PG}_{0} . \quad A s E_{p}$ is more sperialized only when $\mathrm{PG}_{0}$ contains at least one axis of order $>2, L_{\mathrm{ST}}$ applies only to such $\mathrm{PG}_{0}$ 's; and then only if $\mathrm{PG}_{0}$ has either one or two inequivalent sets of three mutually perpendicular axes of even order. More than two such sets cannot occur in any $\mathrm{PG}_{0}$, at least one such set must occur for all cu'sic $\mathrm{PG}_{0}$ 's.

$L_{01}$ we define as a liberalization to a triaxial ellipsoid (including $L_{S}$ if $\mathrm{PG}_{0}$ has axis of order $>2$ ) with one orientational degree of freedom. $L_{01}$ must lead to one of the following subgroups: $2, \mathrm{~m}$, or $2 / \mathrm{m}$. From any $\mathrm{PG}_{0}$ there will be as many distinct ways of applying the $L_{01}$ operation as there are nonequivalent nonparallel axes of even order symmetry in $\mathrm{PG}_{0}$. Cubic point-groups have at least one and never more than two such directions. Noncubic $\mathrm{PG}_{0}$ may have none, one, two, or at most, three such directions. Nonequivalent $L_{01}$ operations on any centrosymmetric $\mathrm{PG}_{0}$ must all lead to $2 / \mathrm{m}$, but for noncentrosymmetric $\mathrm{PG}_{0}$ 's they may or may not lead to the same subgroup.

$L_{02}$ is a liberalization to a triaxial ellipsoid with two orientational degrees of freedom; the operation is regarded as including an $L_{S}$ operation from $\mathrm{PG}_{0}$ 's with an axis of order $>2$. Application of $L_{02}$ to centrosymmetric $\mathrm{PG}_{0}$ 's always produces the subgroup $\overline{1}$

In this discussion symmetry axes may be rotation or inversion axes. and none other, noncentrosymmetric $\mathrm{PG}_{0}$ 's lead only to 1 .

The aim of the treatment here given is to consider the symmetry and orientation of $E_{p}$; then to apply the liberalizations, $L$, until the symmetry and orientation of $E_{s}$ is obtained. This process cannot always be accomplished because $E_{s}$ may in some respects be more restricted than can be derived from $E_{p}$ by $L$-type operations. We must therefore liberalize $E_{s}$ to $E_{s}^{\prime \prime}$ where $E_{s}^{\prime}$ is a more general ellipsoid which can be reached from $E_{p}$ by operations $L$ and which will produce the same symmetry lowering as $E_{s}$.

If $E_{s}$ is a triaxial ellipsoid it is in an adequately general form because it can be obtained from any ellipsoid by operations $L_{\mathrm{ST}}, L_{01}$, or $L_{02}$. If $E_{s}$ is a biaxial stress, one need only add an arbitrary isostatic stress to obtain an acceptable $E_{s}^{\prime}$. It has previously been pointed out [10] that the addition of such an isostatic stress can not affect the symmetry lowering. The on! $\mathrm{y}$ important case to be considered therefore is that of a stress $E_{s}$ corresponding to an ellipsoid of revolution, or a uniaxial stress which by the addition of an arbitrary isostatic stress can be transformed to one corresponding to an ellipsoid of revolution. We must now distinguish two possible conditions as follows:

Condition 1: unique ellipsoid axis is parallel to a crystal axis (in $\mathrm{PG}_{0}$ ) of order $>2$. The symmetry of $E_{s}$ can not be further reduced without affecting the symmetry lowering. No further generalization of $E_{s}$ is therefore possible.

Condition 2: unique ellipsoid axis is parallel to a crystal axis (in $\mathrm{PG}_{0}$ ) of order $\leq 2$. There is now necessarily a uniaxial stress which can be added perpendicular to the unique $E_{s}$ ellipsoid axis which will leave the symmetry lowering unaffected. If in $\mathrm{PG}_{0}$ there is no even order axis perpendicular to the unique ellipsoid axis the direction of the additional uniaxial stress is entirely arbitrary. If there are one or two perpendicular directions of axial even order $\geq 2$ in the plane perpendicular to the unique ellipsoid axis the additional uniaxial stress must be added parallel to one axis in the plane.

Our general scheme of deriving all subgroups can now be stated.

All proper subgroups are developed by operations $L_{\mathrm{SR}}, L_{\mathrm{ST}}, L_{01}$, and $L_{02}$, each in turn considered as acting upon $\mathrm{PG}_{0}$.

The number of proper subgroups obtained is:

\begin{tabular}{|c|c|c|c|c|c|c|}
\hline & $L_{\mathrm{SR}}$ & $L \mathrm{sT}$ & $L_{01}$ & $L_{02}$ & $\begin{array}{l}\text { Maximum } \\
\text { number } \\
\text { possible }\end{array}$ & Example \\
\hline $\mathrm{PG}_{0}$ cubic $\ldots$ & 1 or 2 & 1 or 2 & 1 or 2 & 1 & 7 & $\mathrm{~m} 3 \mathrm{~m}$ \\
\hline $\mathrm{PG}_{0}$ one and only one axis order $>3 \ldots$ & 0 & 0,1 , or 2 & 1,2 , or 3 & 1 & 6 & $4 / \mathrm{mmm}$ \\
\hline $\begin{array}{l}\mathrm{PG}_{0} \text { one and only one axis order }=3 \text { and } \\
\text { none of higher order }\end{array}$ & 0 & 0 & 0 or 1 & 1 & 2 & $\overline{3} \mathrm{~m}$ \\
\hline $\begin{array}{l}\mathrm{PG}_{0} \text { with axis order } 2 \text { and none of higher } \\
\text { order }\end{array}$ & 0 & 0 & 1 or 3 & 1 & 4 & $\mathrm{mmm}$ \\
\hline
\end{tabular}


The naming of the individual subgroups is trivial when we remember that a center in $\mathrm{PG}_{0}$ is always retained; that for $L_{\mathrm{SR}}$ the first symbol is derived from the only axial direction parallel to the ellipsoid axis of revolution. The necessary and sufficient condition of 2-axes retention is then that they be parallel or perpendicular to that direction; that for $L_{\mathrm{ST}}$ only the crystal axes (order 2) parallel to the principal ellipsoid axes are retained; that for $L_{01}$ only one axial symmetry direction (order 2) can be retained.

Minimum steps are produced by all $L_{\mathrm{SR}}$ operations; by all $L_{\mathrm{ST}}$ operations except when $\mathrm{PG}_{0}$ is cubic and has three axes of order 4 ; by $L_{01}$ operations when the operation $L_{\mathrm{ST}}$ on $\mathrm{PG}_{0}$ is inapplicable; and by $L_{02}$ operations only if $\mathrm{PG}_{0}$ has only one axial direction of order two or three.

The authors are indebted to F. A. Munley and L. C. McCleary for help in the preparation of the manuscript as well as especially to W. F. Stancliff for the drawing of the stereograms.

\section{References}

[1] J. F. Nye, Physical properties of crystals, Oxford University Press (1957).

[2] V. A. Koptsik, Polymorphic Phase Transitions and Symmetry, Soviet Physics Crystallography 5, 889 (1961). Translated from Kristallografiva 5, 932 (1960).

[3] J. B. Wachtman, Jr., and H. S. Peiser, Symmetry conditions for internal friction caused by jumping of point defects in crystals, App. Phys. Letters 1, 20 (1962)

[4] R. L. Aggorwal and A. K. Ramdas, Effect of uniaxial stress on the excitation spectra of donors in silicon, Phys. Rev. 13\%, A 602 (1965).

[5] Elsa Rosenvasser Felker, Effect of uniaxial stress on the paramagnetic spectra of $\mathrm{Mn}^{3+}$ and $\mathrm{Fe}^{3+}$ in $\mathrm{MgO}$, Phys. Rev. 136, A 145 (1964).
[6] H. S. Peiser, J. B. Wachtman, Jr., and R. W. Dickson, Reduction of space groups to subgroups by homogeneous strain, J. Research NBS 67A, 395 (1963).

[7] A. S. Nowick and W. R. Heller, Anelasticity and stressinduced ordering of point defects in crystals, Adv. in Phys. 12, 251 (1963).

[8] J. B. Wachtman, Jr., H. S. Peiser, and E. P. Levine, Symmetry splitting of equivalent sites in oxide crystals

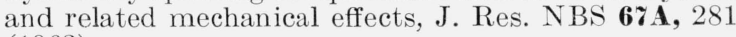
(1963).

[9] A. S. Nowick and W. R. Heller, Dielectric and anelastic relaxation of crystals containing point defects, Adv. in Phys. 14, (1965).

[10] J. B. Wachtman, Jr. and H. S. Peiser, Splitting of a set of equivalent sites in centrosymmetric space groups into subsets under homogeneous stress, J. Res. NBS 69A, No. 2, 193-207 (1965).

[11] N. F. M. Henry and Kathleen Lonsdale, editors, International tables for x-ray crystallography, Vol. I, Kynoch Press, Birmingham (1952).

[12] G. F. Koster, Space groups and their representations, Solid State Physics, Vol. 5, pages 173-256, Edited by F. Seitz and D. Turnbull, Academic Press, New York (1957).

[13] F. Seitz, A matrix-algebraic development of the crystallographic groups, Z. Krist. 88, 433 (1934); 90, 298 $(1935) ; \mathbf{9 1}, 336(1935) ; \mathbf{9 4}, 100(1936)$.

[14] H. S. Peiser, J. B. Wachtman, Jr., F. Munley, and Linda C. MeLeary, Splitting of a set of equivalent points in non centrosvmmetric space groups in to subsets under homogeneous stress, J. Res. NBS $\mathbf{6 9 A}$, No. 5 (1965).

[15] F. C. Phillips, An Introduction to Crystallography (Wiley, N.Y., 1964).

[16] M. J. Buerger, Crvstallographic aspects of phase transformations, pp. 183-211 in Phase Transformations in Solids, edited by R. Smoluchowski, J. E. Mayer, and W. A. Weyl (John Wiley \& Sons, New York, N.Y., 1951).

[17] A. P. Honess, The Nature, Origin, and Interpretation of Etch Figures on Crystals (John Wiley, N.Y., 1927).

[18] A. V. Shubnikov, N. V. Belov, and others, "Colored Symmetry" Translated from the Russian by J. Itzkoff and $\boldsymbol{J}$. Gollob; Edited by W. T. Holser, Pergamon Press (1964).

(Paper 69A4-351) 